\title{
TV/Series
}

$4 \mid 2013$

Écho et reprise dans les séries télévisées (II) : Re-

présentations -- enjeux socio-culturels, politiques et idéologiques de la reprise

\section{Friday Night Lights (NBC et The 101 Network, 2006-2011) ou le réinvestissement du mythe de la small town}

\section{Gérald Billard et Arnaud Brennetot}

\section{OpenEdition \\ Journals}

Édition électronique

URL : http://journals.openedition.org/tvseries/739

DOI : $10.4000 /$ tvseries.739

ISSN : 2266-0909

Éditeur

GRIC - Groupe de recherche Identités et Cultures

\section{Référence électronique}

Gérald Billard et Arnaud Brennetot, «Friday Night Lights (NBC et The 101 Network, 2006-2011) ou le réinvestissement du mythe de la small town», TV/Series [En ligne], 4 | 2013, mis en ligne le 15 décembre 2013, consulté le 19 avril 2019. URL : http://journals.openedition.org/tvseries/739 ; DOI : $10.4000 /$ tvseries.739

\section{cc) (†)}

TV/Series est mis à disposition selon les termes de la licence Creative Commons Attribution - Pas d'Utilisation Commerciale - Pas de Modification 4.0 International. 


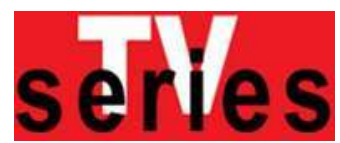

Friday Night Lights ${ }^{1}$ ou le réinvestissement du mythe de la small town

Gérald BILLARD

Arnaud BRENNETOT

À partir de l'exemple de Friday Night Lights, cet article entend montrer comment la double adaptation d'un livre documentaire puis d'un film au format d'une série télévisée peut conduire à une amplification et à un approfondissement de la mise en scène tels que l'histoire particulière d'une petite communauté locale du Texas conduit à une relecture originale de quelques-uns des grands thèmes de la fiction télévisée contemporaine (la réalisation de soi, la cohésion sociale, la pression communautaire, etc.). Le réinvestissement du mythe géographique de la small town donne alors l'occasion aux créateurs de la série de proposer une représentation humaniste et nuancée de la vie collective, alternative aux visions désabusées ou nihilistes qui prolifèrent par ailleurs dans nombre de drama series diffusées sur les chaînes du câble aux États-Unis, sans pour autant renoncer à la vraisemblance des événements ou à la puissance des émotions.

$\mathrm{L}$ es séries télévisées adaptées d'œuvres antérieures semblent être devenues une forme récurrente de la production sérielle étatsAnienne contemporaine (voir figure 1). Les enjeux de la transposition diffèrent néanmoins fortement selon qu'il existe une homologie entre le format narratif des œuvres transposées et celui du support d'adaptation. Le remake ou le reboot d'une série préexistante ne représente pas les mêmes contraintes que l'adaptation d'un film, dont la durée est forcément limitée, au format plus long d'une série télévisée, appelée à se déployer sur une ou plusieurs saisons. Dans ce second cas, l'adaptation ne peut pas être une simple transposition mais exige un étirement et un enrichissement du récit, le développement de certains thèmes inédits, l'invention de personnages supplémentaires, le recours à des flashbacks ou à des flashforwards.

$\mathrm{La}$ réinvention du récit qu'occasionnent de telles transformations peut aller très loin, au point même d'aboutir parfois à des écarts, des torsions ou des inversions du discours présent dans l'œuvre originelle (scénario, genre esthétique, teneur morale, etc.). Toute adaptation s'apparente alors à un ensemble de reprises et de transformations, inégalement proportionnées, qui autorisent un jeu permanent avec les téléspectateurs autour de la reconnaissance des points communs et des différences.

${ }^{1}$ NBC et The 101 Network, 2006-2011. 


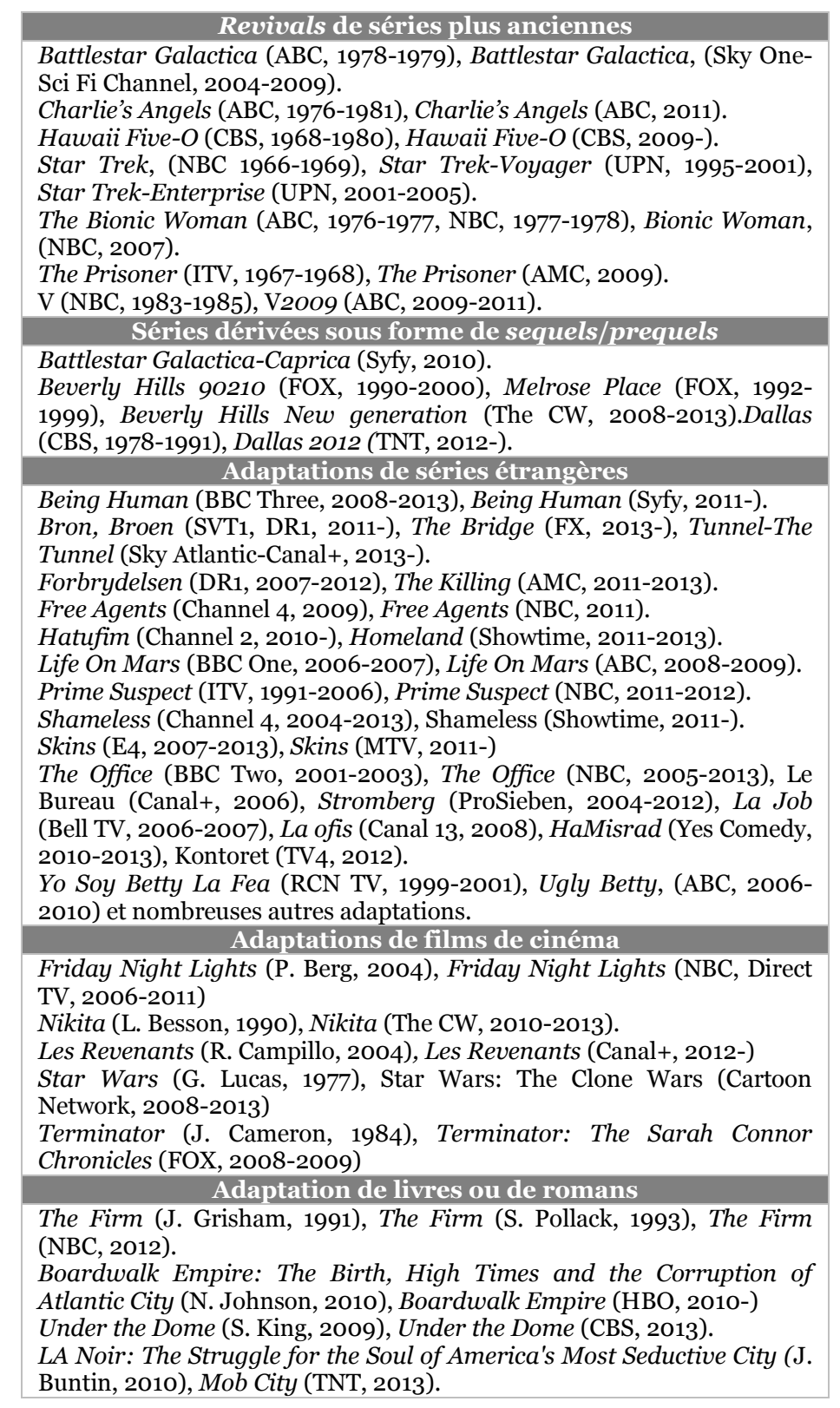


Adaptations de séries de romans

A Song of Ice and Fire (G. R. Martin, 1996-), Games of Thrones (HBO, 2011-)

Darkly Dreaming Dexter (J. Lindsay, 2004-2011), Dexter (Showtime, 2006-2013).

The Southern Vampire Mysteries (C. Harris, 20o1-), True Blood (HBO, 2008-)

Roswell High (M. Metz, 1998-2000), Roswell (The WB, 1999-2001, UPN, 2001-2002).

Walt Longmire (C. Johnson, 2004-), Longmire (A\&E, 2012-)

The Sword of Truth (T. Goodkind, 1994-2013), Legend of the Seeker (ABC, 2008-2010).

Adaptations de séries en bandes dessinées

Smallville (DC Comics, 1949), Smallville (The WB, 2001-2006, The CW, 2006-2011)

The Walking Dead (R. Kirkman, 2003-), The Walking Dead (AMC, 2010-)

Fig. 1 : Exemples de reprises dans les séries télévisées aux États-Unis

Dans ce contexte, les lieux diégétiques se présentent souvent comme des éléments de stabilité ${ }^{2}$, facilitant l'identification des liens de descendance et des continuités entre l'œuvre initiale et l'œuvre dérivée : le Deep South pour The Walking Dead et True Blood, le ranch des Ewing pour Dallas, l'archipel hawaïen pour Hawaï Five-O, les grandes villes dans $V$ ou des vaisseaux géants Battlestar Galactica, ou encore Star Trek. Dans la série Game of Thrones, par exemple, le monde minutieusement cartographié dans le générique reprend l'espace diégétique des sept royaumes inventé par George R. Martin pour son cycle romanesque. Lorsqu'il s'agit d'une transposition internationale, les lieux changent mais l'ambiance géographique demeure souvent: les villes de Copenhague et de Seattle pour The Killing, des quartiers populaires de Manchester et de Chicago pour Shameless, des lycées à Bristol et à Baltimore pour Skins, des bureaux impersonnels pour The Office ou des établissements de mode à Bogotà et à New York pour Yo Soy Betty La Fea et Ugly Betty, etc.

Cette permanence est cependant rarement complète et des glissements peuvent s'opérer dans la façon de figurer les lieux et de les intégrer au sein de la trame dramatique. Par exemple, la représentation de la figure idéal-typique de la small town dans les trois versions de Friday Night Lights, le livre, le film et la série, diffère sensiblement : outre le passage du lieu réel (Odessa, Texas) au lieu fictif (Dillon, Texas), la fonction sociale du territoire change profondément, le

2 Il en va différemment pour les franchises (CSI, CBS, 2000-, NCIS, Stargate, Showtime, SyFy, 1997-2011, Law and Order, NBC, 1990-2010, Spartacus, Starz, 2010) où la délocalisation géographique devient souvent, au contraire, un élément de renouvellement, dans un cadre dramatique qui change par ailleurs souvent très peu. Voir par exemple Hélène Monnet-Cantagrel, " CSI, de la série au jeu : 'a more immersive experience' ", TV $/$ Series, $\mathrm{n}^{\circ} 3$, septembre 2013 
passage à la série télévisée révélant une profondeur que les formats du livre documentaire ou du film cinématographique n'avaient pas permis d'atteindre. Cet article propose de montrer comment l'adaptation à la forme sérielle a permis une exploration plus profonde de l'« espace vécu 3 » des personnages, laissant deviner des sensibilités géographiques plus complexes et subtiles que dans les œuvres originelles.

\section{D’Odessa à Dillon : un livre, un film, une série}

Friday Night Lights est initialement un ouvrage écrit en 1990 par Henry Bissinger, journaliste au Philadelphia Inquirer. Ce livre, sous-titré $A$ Town, a Team and a Dream raconte la saison sportive des Permian Panthers, l'équipe de football du lycée de la ville d'Odessa (90 000 habitants), située à l'Ouest du Texas, au cours de l'année 19884. Le livre narre les différents épisodes d'une saison sportive haletante liée à une succession de rebondissements qui conduisent l'équipe en demi-finale du Championnat du Texas. À l'époque, l'auteur choisit de se rendre à Odessa car l'équipe des Permian Panthers possède une longue tradition de victoires au championnat de l'État (1965, 1972, 1980, 1984), laissant deviner l'importance que le football est susceptible de représenter pour la population locale. Au-delà du récit des événements liés au championnat, l'objectif premier du journaliste consiste à dresser une chronique de la vie ordinaire dans une petite ville de l'Amérique profonde, saisie à travers le prisme du football lycéen :

The goal had never been to write a sports book, per se, but rather to take an ethnographic look at life in an American town in which everything was touched by high school football. [...] Small-town life is intricate and gripping and the glue that holds it together is footballs.

Le journaliste s'attache ainsi à décrire avec minutie le contexte sociogéographique dans lequel évoluent les joueurs, en évoquant notamment les divers problèmes qu'ils sont susceptibles de rencontrer à Odessa : la pauvreté, le racisme, la ségrégation et les inégalités sociales. L'ouvrage adopte volontiers une tonalité critique à l'égard d'une ville présentée comme un territoire en décrépitude. La ville, dont l'âge d'or lié à l'exploitation pétrolifère est révolu, ne semble plus avoir désormais d'autre espoir que la réussite de l'équipe du football du lycée pour maintenir sa cohésion interne. Le livre est

3 Armand Frémont, La Région, espace vécu, Paris, Flammarion, 1976.

4 Henry Bissinger, Friday Night Lights, A Town, a Team and a Dream, Cambridge Ma, Da Capo Press, 1990.

5 Interview donnée par Henry Bissinger, dans Sports Illustrated, 14 février 2011. 
d'ailleurs plutôt mal accueilli à Odessa lors de sa sortie alors qu'il connaît un grand succès dans le reste des États-Unis ${ }^{6}$.

Dès 1993, le livre Friday Night Lights, A Town, a Team and a Dream inspire plusieurs scénaristes et fait l'objet d'une première adaptation télévisée : diffusée sur NBC, la mini-série Against the Grain (1993) reprend ainsi de nombreux thèmes abordés dans le livre. Par la suite, en 2004, la trame narrative de Friday Night Lights est réinvestie une deuxième fois sous la forme d'une adaptation cinématographique dirigée par Peter Berg, cousin de Henry Bissinger. Le film reprend le titre du livre (voir figure 2) et respecte fidèlement la trame des événements décrits dans le livre ainsi que l'identité des personnages et des lieux : l'histoire se déroule toujours en 1988 et Odessa continue à apparaitre comme une ville moyenne de l'Amérique profonde, majoritairement habitée de Blancs, de Noirs et de Latinos appartenant aux classes populaires de la société américaine.
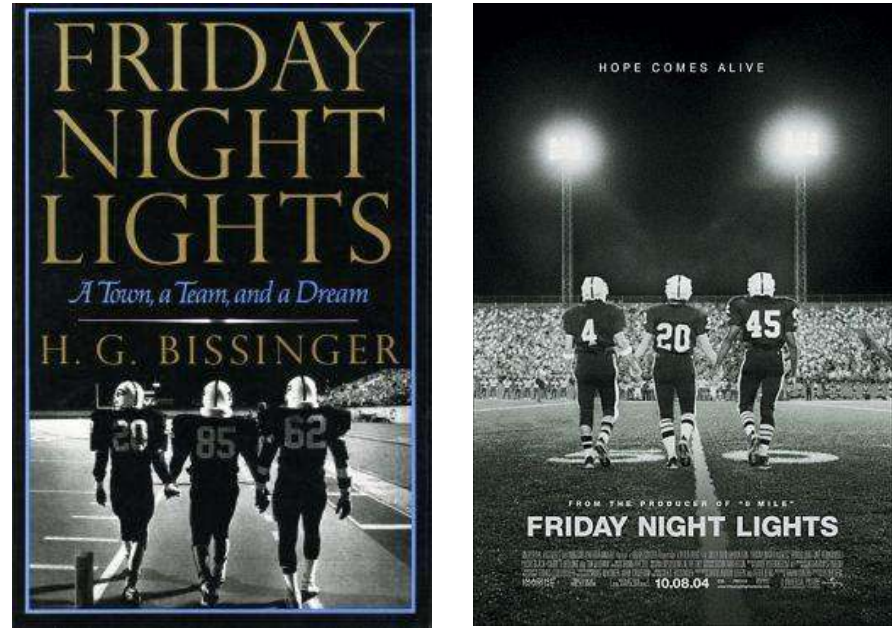

Fig. 2 : Couverture de l'ouvrage et affiche du film Friday Night Lights.

${ }^{6}$ En 2002, Sports Illustrated a désigné le livre Friday Night Lights quatrième meilleur livre de tous les temps consacré au sport.

http://sportsillustrated.cnn.com/si_online/features/2002/top_sports_books/1/ (dernier accès en décembre 2013). 
Comme le livre, le film s’intéresse à certains pans de la vie quotidienne de quelques personnages : le coach, Gary Gaines, joué par Billy Bob Thornton, le fullback, Don Billingsley, ou le running back, Boobie Miles. Le récit demeure pourtant centré sur la façon dont les joueurs envisagent leur rapport au football. L'intrigue principale repose notamment sur les conséquences pour l'équipe de football de la blessure de Boobie Miles, le running back prodige sur lequel toute la ville compte pour remporter le championnat. Avec cette blessure, qui survient lors de la phase de qualification, la poursuite du championnat paraît compromise et les Permians ne doivent leur accès aux playoffs qu'à un heureux tirage au sort contre une équipe adverse parvenue au même nombre de points au terme de la phase de poule.

La passion locale pour le football est telle que Boobie va jusqu'à remettre en cause le diagnostic du médecin de Midland, ville de l'une des équipes rivales, accusant le praticien de participer à un complot contre les Permians afin de les empêcher d'accéder à la finale. Pour beaucoup de joueurs, la réussite sportive constitue un enjeu crucial, notamment car elle représente, pour certains d'entre eux (comme Chavez), la clé pour accéder à l'Université l'année suivante et sortir d'Odessa, ou, pour les moins chanceux (comme Don ou Mike), l'occasion de connaître un ultime moment de gloire avant d'entamer une vie d'adulte morne et précaire, à l'image du père de Don Billingsley. Cet échange entre Don, Mike et Chavez montre bien l'importance relative que la réussite au football représente pour leur avenir :

MiKE. He designed his offence around one player. We're dead.

CHAVEZ. We're not dead. We just need to start throwing the football.

Don. We're dead.

Mike. Dead. Pull it.

CHAVEZ. We gotta lighten up. We're seventeen.

DoN. Do you feel seventeen?

MiKe. I don't feel seventeen. Pull it.

CHAVEZ. We will win state.

Don. Chavo, you're like a humana piñata. You get your ass beat on more than anybody I know and you just sit there and spit out candy.

MiKE. That's cause he's out of here. He's got the grades. No matter what, if we win or lose, he knows he's getting out. He's like the one foot out the door, man.

DON. You'll be drinking martinis, eating lamb chops, getting manicures. CHAVEz. You're just jealous.

Dans le film, le coach Gaines se présente comme une figure paternelle, dont l'horizon paraît plus ouvert que le reste de la population d'Odessa et qui, de ce fait, pousse les joueurs à se surpasser et à donner le meilleur d'eux-mêmes. Le championnat s'apparente alors à une épreuve initiatique et collective, censée permettre à chaque joueur de sortir grandi, malgré la défaite en demi-finale, et de se 
préparer à affronter les futures épreuves de la vie. Le film Friday Night Lights reprend ainsi bon nombre des codes du sports movie, bénéficiant de critiques plutôt positives lors de sa sortie7.

Après un succès en salles relativement modeste, Peter Berg décide l'année suivante d'adapter Friday Night Lights en série télévisée, s'adjoignant pour cela la collaboration de Jason Katims, le producteur de la série Roswell. Lancée en 2006, la série Friday Night Lights a été diffusée pendant cinq saisons, chacune d'elle racontant une année de championnat. Diffusée initialement par NBC, comme Against the Grain précédemment, la série réunit entre cinq et neuf millions de téléspectateurs ${ }^{8}$, audience suffisamment réduite sur un network pour que le diffuseur menace de ne pas la reconduire à l'issue de la deuxième saison. Elle ne doit son prolongement au-delà qu'à un accord de partenariat signé avec le bouquet de diffusion par satellite Direct TV qui programme la série sur la chaîne The 101 Network jusqu'en 2011.

D'un point de vue narratif, la série Friday Night Lights partage avec le film de nombreux points communs : les ambiances visuelle et sonore particulièrement soignées, les maisons filmées à l'identique, les grands espaces avec les routes qui se perdent à l'horizon, les paysages emblématiques du Texas rural, les puits de pétrole plus ou moins en activité, les réservoirs d'eau, la présence quotidienne de la radio locale, sans oublier la musique aérienne du groupe post-rock Explosions in the Sky, originaire d'Austin, où la série est tournée (voir figures 4 et 5).

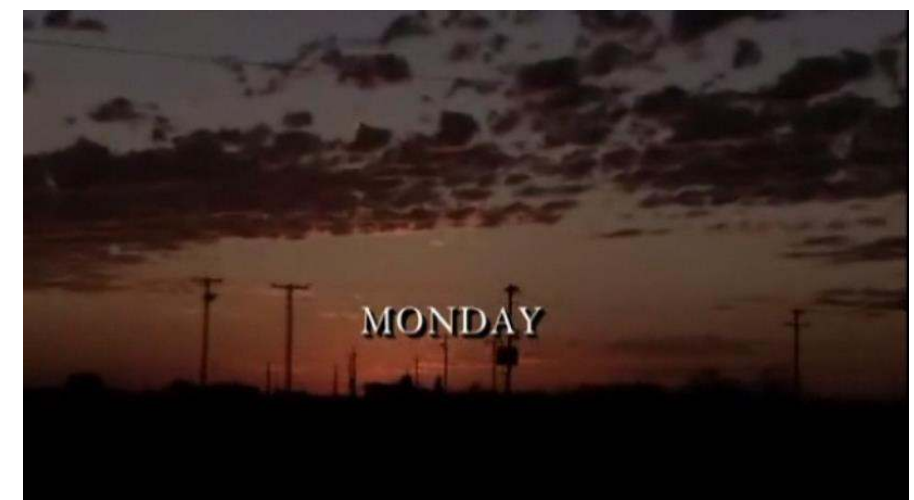

Fig. 4 : Lever de soleil sur Dillon, première image du pilote de la série Friday Night Lights.

\footnotetext{
7 Voir http://www.metacritic.com/movie/friday-night-lights/critic-reviews (dernier accès en décembre 2013).

8 Voir : http://friday-night-lights.hypnoweb.net/serie/audiences.104.363/ (dernier accès en décembre 2013).
} 


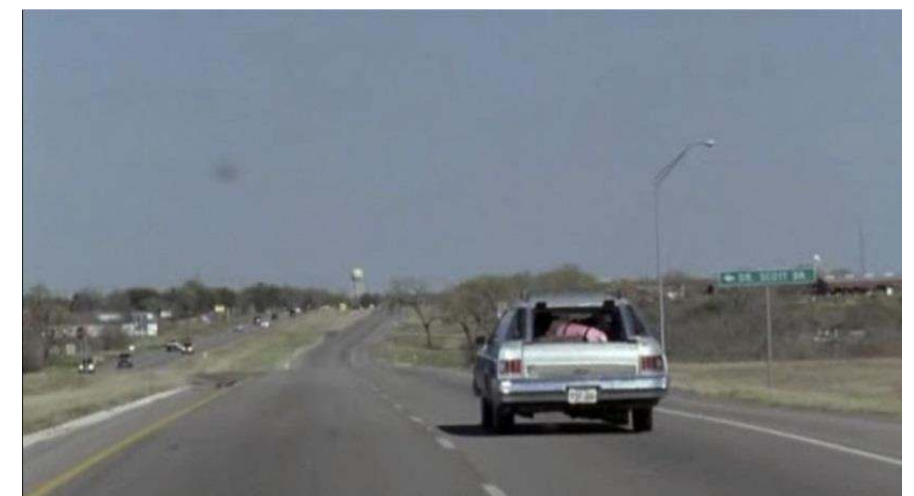

Fig. 5 : Les grands espaces du Texas où l'automobile demeure omniprésente

Les créateurs de la série ont cependant profité de l'adaptation au format télévisuel pour introduire plusieurs différences scénaristiques importantes. La série se déroule ainsi dans une petite ville fictive de l'Ouest du Texas, baptisée Dillon, sans doute un peu moins peuplée qu'Odessa. En outre, si les championnats de football représentent un fil narratif qui structure chaque saison, ils n'occupent toutefois plus la majorité des scènes. La série s'intéresse plus directement à la vie quotidienne des joueurs, observe ce qui se passe dans leur entourage, dans leur famille ou au lycée. Une place importante est également accordée aux personnages extérieurs à l'équipe de football, notamment aux personnages féminins. Les personnages principaux de la série ne sont pas les joueurs mais le couple formé par le coach Taylor et sa femme, Tami, jouée par l'actrice Connie Britton, comme dans le film. Beaucoup de scènes s'attachent à les suivre en famille avec leurs deux filles, Julie et Gracie Belle, mais aussi dans la petite ville de Dillon, notamment au lycée et dans le stade. Les autres arcs narratifs concernent la vie quotidienne des joueurs de l'équipe mais aussi des cheerleaders ou des autres camarades du lycée, comme Tyra Collette ou Landry Clarke. La prépondérance des intrigues parallèles à la compétition sportive fait que la série Friday Night Lights ne correspond pas tout à fait à un sports drama traditionnel. De même, en raison de la place réservée aux adultes, Friday Night Lights n'est pas non plus une teen series au sens strict.

\section{Un réalisme au service d'une vision humaniste des relations sociales}

Si la série est difficile à rattacher à un genre particulier, elle témoigne aussi de la recherche d'une certaine authenticité dans la scénarisation et dans la mise en scène. Les épisodes ont notamment été 
tournés dans des décors naturels, au Texas, à la périphérie d'Austin, ce qui permet aux réalisateurs de créer un effet de réalisme rarement utilisé dans le reste de la production télévisuelle états-unienne.

La série s'emploie en particulier à filmer les « non-lieux » de la vie quotidienne, les stades, les bordures de route, les carrefours, les parkings impersonnels ou les fast-foods éclairés au néon (voir figure 6). La série donne ainsi à voir tout ce qu'il peut y avoir d'inachevé dans les paysages ordinaires que les personnages habitent, marquant leur présence de multiples signes: des drapeaux, des bannières ou des écriteaux mais aussi la décoration des intérieurs domestiques, le mobilier et tous les objets qui emplissent la vie quotidienne.
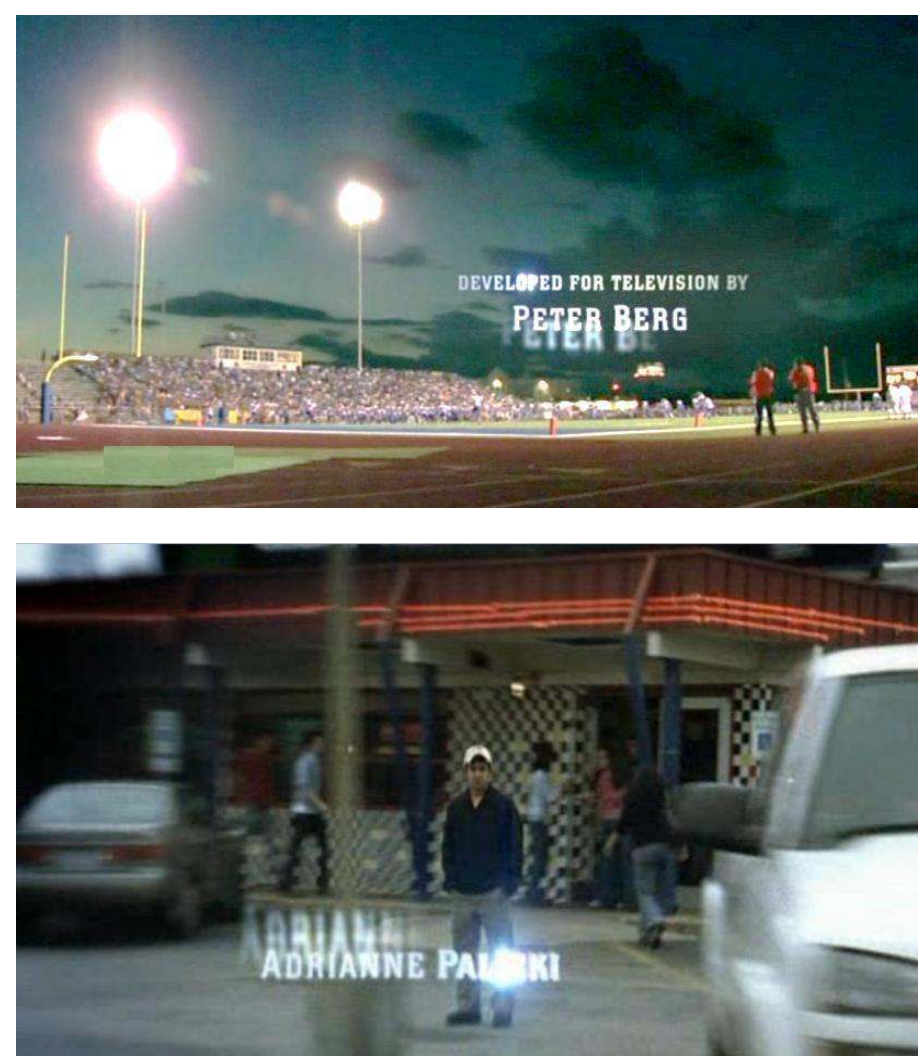

Fig. 6 : Le stade et le fast-food - lieux emblématiques de la vie ordinaire dans l'Amérique profonde (générique de la série Friday Night Lights).

La série est tournée comme un véritable documentaire, avec des éclairages naturels, une grande liberté accordée aux acteurs, des 
cadrages apparemment approximatifs qui donnent l'impression de scènes saisies sur le vif. S'ajoutent en outre de nombreuses scènes contemplatives filmées en plans larges, captant les paysages de la nature et du ciel texans, et des moments où l'on observe les personnages mener leurs activités, sans qu'on puisse les entendre parler. On saisit alors l'expression des corps et des visages dans leur intimité, comme si les personnages n'étaient pas joués ou comme si la caméra n'était pas là. Loin d'être oniriques, ces scènes contemplatives renforcent le caractère réaliste de la série, les moments non dialogués conférant une présence physique très forte aux personnages comme aux paysages.

Cette manière de filmer contribue à l'équilibre narratif de la série, la plupart des épisodes alternant des phases spectaculaires et excitantes consacrées au déroulement des matchs et des moments plus calmes, où l'on suit les personnages dans l'intimité de leur vie quotidienne.

Le réalisme de Friday Night Lights tient aussi aux nombreuses intrigues qui abordent divers problèmes de la vie réelle: le délaissement familial et la faillite parentale, la pauvreté, le racisme et la ségrégation, la délinquance juvénile, la dépendance et la solitude ou les défaillances du corps (handicap moteur, maladie d'Alzheimer). Les lieux que les personnages habitent reflètent eux aussi la réalité, parfois dure, des inégalités sociales : le ghetto noir dans lequel habitent Smash Williams (Saisons 1 et 2) ou Vince Howard (Saisons 4 et 5), la bicoque défraîchie de la grand-mère de Matt Saracen (voir figure 7), la maison middle class des familles Taylor et Garrity, la mansion ostentatoire des MacCoy, la caravane vétuste dans laquelle Tim Riggins s'est réfugié et même le lycée et le stade délabrés d'East Dillon à partir de la quatrième saison.

Les adolescents de Friday Night Lights n'échappent pas aux périls d'une vie chaotique et beaucoup souffrent directement et durablement des multiples drames qui leur arrivent. Jason Street, le quarterback vedette de l'équipe, est ainsi condamné, après un accident survenu dès le pilote, à passer sa vie dans un fauteuil roulant. Certains joueurs sont menacés par des gangs ou surveillés par la police, et finissent par se retrouver en prison pour des délits plus ou moins graves. Beaucoup souffrent par ailleurs des défaillances familiales, du délaissement, de la fuite ou de la mort d'un parent. Pour plusieurs, la pauvreté économique contrarie leurs chances de voir se réaliser leurs projets d'avenir. Si les garçons subissent la pression permanente du modèle masculin du compétiteur sportif, il n'est pas rare que les lycéennes soient de leur côté victimes de l'intolérance et des préjugés sociaux concernant la sexualité féminine. 

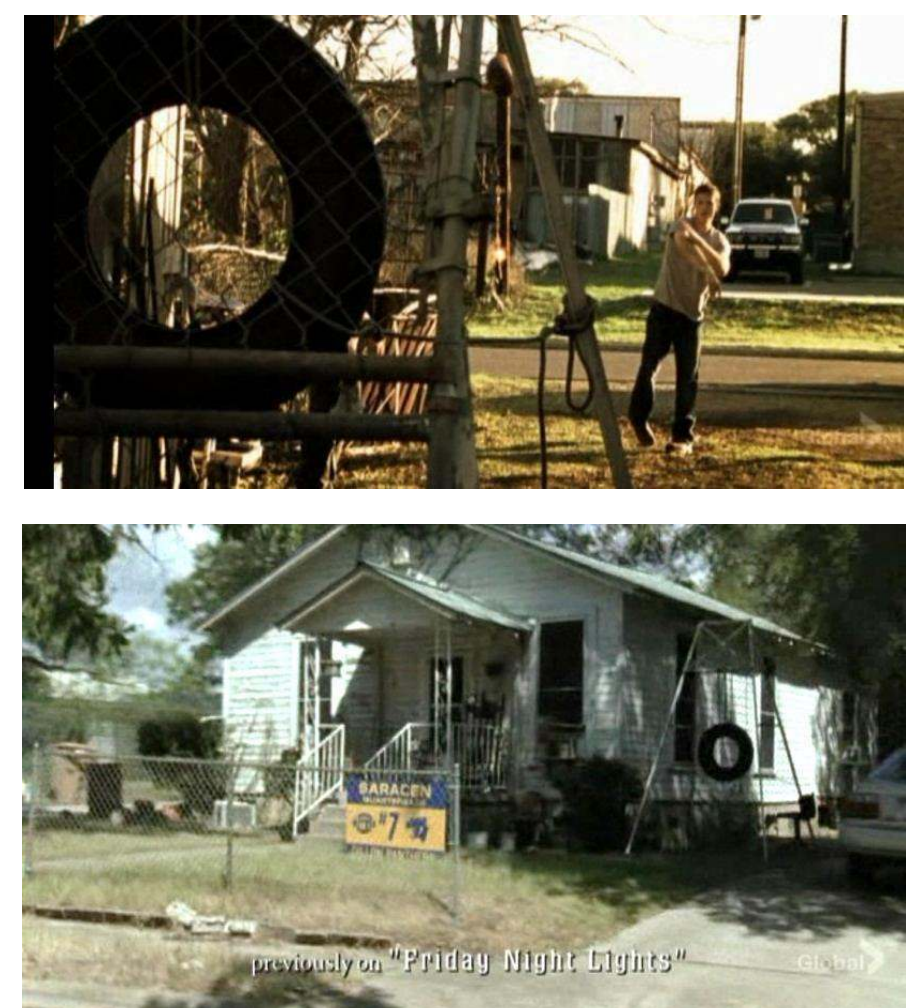

Fig. 7 : La maison de la grand-mère de Matt Saracen, située dans un quartier habité par la classe populaire blanche (Friday Night Lights, 1.4).

Toutes ces difficultés rendent plus touchants les divers moments de bonheur et de réussite que les adolescents connaissent : la victoire d'un match, l'admission à l'université, la réussite professionnelle, le plaisir de jouer dans un garage band, le début d'une liaison amoureuse ou, plus tard, la joie de fonder une famille. Cependant, ces réussites ne sont que des succès provisoires, des conquêtes personnelles qui ne préjugent en rien de l'avenir. Parce qu'elle évite ainsi le double écueil du misérabilisme et de l'angélisme, Friday Night Lights est une série dramatique gracieuse et émouvante. C'est bien car elle n'est ni abusivement optimiste, ni excessivement pessimiste, que les moments d'émotion n'en sont que plus authentiques et appréciables?.

9 À titres d'exemples, on citera la célébration de la victoire à la fin de la première saison (1.22) ou les funérailles du père de Matt Saracen (4.5). 
Dans la série Friday Night Lights, si la mise en scène vise à laisser transparaître une certaine authenticité, la description de la réalité sociale ne sombre jamais dans le fatalisme, notamment car, à Dillon, l'espérance n'est jamais dérisoire. Les créateurs entendent ainsi montrer qu'il est possible de faire des choix responsables, parfois rendus difficiles en raison des contraintes sociales, mais des choix capables de changer la vie de l'entourage des personnages. La solidarité qui en découle ne parvient certes jamais à supprimer totalement les drames de la vie, y compris ceux qui résultent des diverses formes de précarité sociale, mais elle permet de faire en sorte que les individus, les jeunes en l'occurrence, puissent eux aussi accéder à la dignité et que leurs efforts pour réaliser leurs projets de vie ne soient pas vains. La crédibilité des arcs narratifs tient aussi au fait que les adultes qui aident ces jeunes, notamment le couple Taylor ou des personnages comme Buddy Garrity ou Mindy Riggins, ne sont pas exempts de défauts et ne sont pas infaillibles, notamment lorsqu'eux-mêmes sont confrontés aux difficultés que traverse leur propre famille.

Plusieurs structures d'encadrement offrent alors aux jeunes la solidarité et la sécurité qui vont leur permettre de grandir, de s'engager et de prendre des risques, pour finir par s'émanciper, devenir pleinement autonomes et accéder à la dignité que la série présente comme la fin ultime de l'existence humaine : le lycée ou l'équipe de football représentent autant de cellules sociales qui favorisent l'expression de ce cheminement initiatique. À Dillon, les communautés religieuses jouent également un rôle très important. Elles apparaissent en filigrane, tout au long de la série, comme un élément structurant de la vie des personnages, sans que soit gommée l'hypocrisie des bons sentiments échangés lors des cérémonies collectives ou les excès de ferveur et d'intolérance que suggèrent certains personnages born again (Lyla Garrity ou la mère de Luke Cafferty, par exemple). Là encore, il n'y a pas de moralisme, ni de manichéisme. On suit les personnages dans leurs efforts pour faire face aux épreuves de la vie, qu'ils réussissent ou qu'ils défaillent, qu'ils soient croyants ou non, que leur famille soit équilibrée ou au bord du chaos.

Parmi les différentes formes communautaires servant de cadre structurant aux individus, il en est une qui apparaît de façon essentielle tout au long de la série : il s'agit de la ville de Dillon elle-même. Pour cette raison, si Friday Night Lights n'est ni une sports series, ni une teen series à proprement parler, nous proposons de la qualifier de small town series. 


\section{La small town: cadre ambigu de l'émancipation individuelle}

\section{Une ville suspendue à l'équipe de football du lycée}

Dès les premiers épisodes de la série, à l'image d'Odessa dans le livre de Henry Bissinger ou dans le film de 2004, la population de Dillon semble entièrement suspendue à la vie de l'équipe de football du lycée. Le vendredi soir, lorsque surviennent les matchs, toute la ville semble se rassembler dans un élan communautaire pour assister à la rencontre sportive. Au cours des jours qui précèdent, les radios locales commentent en boucle les chances des équipes qui vont s'affronter. Les joueurs et le coach sont interviewés et invités à commenter les dernières rumeurs concernant la stratégie de l'entraîneur ou l'état physique de tel ou tel joueur. Dans les bars, les fast-foods ou les fêtes entre amis, toutes les discussions semblent tourner autour des matchs de football. Au tout début de la série, le personnage de Jason Street, le QB1 (quarterback 1) de l'équipe, est présenté comme la grande vedette de Dillon sur laquelle reposent tous les espoirs de la ville. De son côté, Buddy Garrity, un concessionnaire automobile de la ville, est devenu un véritable notable à Dillon en raison du fait qu'il occupe aussi le poste de président de l'amicale des anciens joueurs. En communion avec le reste de la ville, la maire de Dillon ${ }^{10}$ semble elle aussi attacher une grande importance au football : l'encouragement de l'équipe ou la recherche de sponsors semble d'ailleurs occuper une bonne partie de son énergie. L'équipe de football apparaît bien comme le centre d'intérêt principal de toute la ville.

\section{La small town : un piège oppressant pour les adolescents?}

Cette focalisation de la population de Dillon autour de l'équipe de football du lycée se traduit par une très forte pression sur les lycéens, au point de leur être parfois insupportable. L'accident dont Jason Street est victime lors du premier match du championnat, au début de la première saison, annonce l'ambiguïté du rapport des jeunes à la small town. Cet événement tragique pour sa propre existence, le condamnant à se déplacer en fauteuil roulant jusqu'à la fin de ses jours et ruinant sa carrière sportive, compromet aussi les chances de l'équipe et, à travers elle, de la ville tout entière pour le reste du championnat : à sa propre douleur s'ajoute donc le sentiment de culpabilité d'avoir

${ }^{10}$ On notera que le choix d'une femme pour occuper le poste de maire permet aux créateurs de la série de désamorcer l'hypothèse que Dillon pourrait s'intéresser au football uniquement car elle serait une ville de rednecks conservateurs, phallocrates et réactionnaires. 
ruiné les espoirs de la ville. Jason passe ainsi du statut d'icône adulée à celui de figure sacrificielle que la ville a détruite.

Pour Matt Saracen, qui remplace Jason au poste de $Q B 1$, la pression est également difficile à supporter, alors que lui-même doit faire face à une situation familiale particulièrement difficile : "It's a big deal because then this whole town is gonna murder me. And then I'm gonna be sitting on the bench again" (Matt Saracen, 1.19). Loin d'être un loisir ou un moyen de se délasser et d'échapper aux tracas de la vie quotidienne, le football s'impose comme un facteur de stress supplémentaire que la ville impose aux joueurs. Le football n'est pas seulement un jeu. Il semble être au fond la dernière raison qui donne aux habitants de Dillon le sentiment de former une communauté :

BUDDY GARRITY. This is not just some game. You realize that. After what's happened with Jason. I mean, this is about tradition. It's about this town. It's about the spirit and keepin' this town alive. That's football. That's all we got. Now, if we lose this game, this town's just gonna be in a turmoil. And I don't need that.” (1.2)

Dans ces conditions, le football s'impose comme un élément omniprésent et contraignant dans la vie des jeunes : "You don't go against the Panthers in this town and you know that" (Jason Street, 1.12). Pour les filles du lycée, la meilleure façon d'exister aux yeux des autres et d'obtenir une reconnaissance sociale semble se réduire à faire partie de l'équipe de cheerleaders, à l'image de Lila Garrity, fille de Buddy et petite amie de Jason au début de la série. Toute réserve vis-àvis de ce que le football représente pour Dillon risque de passer pour un signe de marginalité. Le mépris que Tyra Collette, lycéenne issue d'une famille pauvre, éprouve pour le football constitue la partie visible de l'abjection qu'elle ressent plus largement pour la ville de Dillon, préfiguration de son désir de fuite vers des horizons lointains. Au cours de la première saison, elle décrit les joueurs de la façon suivante :

TYRA COLLETTE. Just a bunch of overheated jocks, too dumb to know they have no future, fighting over a game that has no meaning, in a town from which there is no escape. (1.5)

Pour elle, le football s'apparente à une activité dérisoire à laquelle les habitants s'accrochent pour mieux oublier le fait qu'ils sont coincés dans un dead end où il ne pourra jamais rien leur arriver de positif.

Contrairement au film, les personnages féminins de la série jouent ainsi un rôle crucial, leur regard souvent décalé permettant de mieux souligner le caractère pathétique du délire sportif dont la ville semble parfois être prisonnière. Tami Taylor, la femme du coach, qui devient également conseillère d'éducation du lycée après leur arrivée dans la ville au début de la première saison, offre un contrepoint significatif. À plusieurs reprises, elle intervient pour mettre en garde son mari contre les illusions que le football pourrait susciter chez les joueurs qu'il entraîne : 
TAMI TAYLOR. You know what the problem is, it's this town, baby. This town makes these teenage kids into idols. And then they get out of here, and everybody's not just giving 'em something all the time. They don't know what to do with themselves. They fall flat on their faces. It's a shame. (1.7)

TAMI TAYLOR. You're making 'em feel like what this town makes 'em feel like, which is that football is the most important thing. (1.11)

Dillon se présente comme l'emblème dystopique des petites villes déclinantes de la périphérie du Texas, à l'instar de nombreuses communes rurales des Plaines de l'Ouest américain, en décalage avec le reste de la société états-unienne ${ }^{11}$, accrochées aux souvenirs des gloires passées.

La small town s'apparente alors à un cadre contraignant pour les jeunes qui y grandissent. Ainsi, si Jason Street se plaint de sa propre condition, ce n'est pas uniquement parce qu'il a perdu l'usage de ses jambes, mais bien aussi car il a l'impression d'être piégé par une ville figée dans le temps, à l'intérieur de laquelle il ne perçoit aucune issue possible :

JASON STREET. Have you ever noticed, that nobody ever changes in this town? Nothing ever changes. All go to the same church, or the same job, and the same restaurants. And everybody goes to the football game Friday night. It's like this huge fishtank, that we're all stuck in and we can't find our way out. I'm kind of... stuck. I'm stuck. (2.6)

À plusieurs reprises, l'expression du désir de fuite s'apparente pour Jason à un cri de désespoir lié au sentiment qu'il ne pourra pas échapper à la vie morne et étriquée que Dillon lui réserve :

JASON. Now pretty much half the town hates me because I'm suing the football team

SuZY. Doesn't that make you wanna get outta there?

JASON. Outta where? Out of Dillon?

SuZY. Yeah! Just leave - screw them!

JASON. Go where? Go where?

SuZY. I don't know... anywhere.

JASON. No, I'm engaged. I can't go anywhere.

SuZY. It's hard for me to understand why anybody would want to be somewhere where they're not wanted.

JASON. It's home. Only home I've ever known. It's just... I can't imagine

I'll be going anywhere else anytime soon. (1.19)

La ville se présente alors comme un horizon fermé qui finit, dans la deuxième saison, par conduire à un rejet et même à une profonde

${ }^{11}$ L'entrée de la société suburbaine états-unienne dans la crise sociale consécutive à l'effondrement du dispositif des subprimes, survenue deux ans après le lancement de la série, atténue un peu ce décalage. Le réalisme social de Friday Night Lights peut même être lu comme l'annonciation anticipée de la fragilité dont le modèle états-unien serait porteur. 
détestation que Jason exprime lors d'une chanson qu'il improvise au cours d'une soirée avec ses amis :

JASON. I'm gonna walk-a, I'm gonna walk-a, I'm gonna get out of my chair, and I'm-a run-a, and I'm-a run-a, and I'm-a get right outta there because Dillon sucks, folks. Dillon is an awful place to be, and I wanna walk away, and tomorrow, I'm gonna do it. (2.4)

À ce titre et à plusieurs reprises, Tyra Collette s'affirme comme le pendant féminin de Jason, elle aussi refusant de se laisser dominer par cette small town dans laquelle le football semble engluer tout un chacun, y compris la femme du coach qui incarne un modèle que Tyra rejette :

TYRA. I don't wanna be you, Mrs. Taylor. I don't wanna stay here. Stuck in this small town... in a job like this... married to a coach. (1.6)

Malgré ses difficultés en mathématiques, l'École devient alors pour la jeune adolescente le seul espoir de sortie, la seule opportunité d'accéder à une université qui lui permettra d'échapper au tropisme de la small town.

\section{Dillon, un tremplin vers l'avenir}

Friday Night Lights ne se résume cependant pas à une satire de la promiscuité locale ou à une simple dénonciation de l'esprit de clocher (parochialism) qui sévirait dans les petites villes de l'Amérique profonde. Parce que ses créateurs cherchent en permanence à éviter tout misérabilisme et toute dénonciation simplificatrice, le discours de la série est bien plus complexe, lui conférant un caractère souvent touchant - certains diraient lacrymal ou édifiant. À Dillon, la réussite et l'espérance ne sont en effet jamais vaines ou insignifiantes.

Tout d'abord, le rejet et la révolte exprimés par certains ados sont tempérés par de nombreux moments où on les voit s'amuser, s'attacher aux autres, tomber amoureux, se lier d'amitié, prendre confiance en eux, se lancer dans des projets, etc. De même, les adultes ne sont pas uniquement montrés comme irresponsables et indifférents à l'égard du sort des autres. À ce titre, le rôle joué par le couple Taylor est souvent crucial pour beaucoup de jeunes : l'espace de proximité qu'offre la petite ville permet aux Taylor d'apporter un soutien réel à tous ces jeunes délaissés par leur famille, tels Matt Saracen, Tyra Collette, Smash Williams, Vince Howard, Becky Sprawles, etc. Dillon n'est plus alors seulement le piège que dénoncent Jason et Tyra : la petite ville devient aussi un cadre territorial qui rend possible le développement de formes inattendues de communication, de sympathie et de solidarité. La proximité géographique favorise ainsi la multiplication des rencontres intersubjectives et devient propice à la communication et à la responsabilisation morale des individus. Ce qu'Eric Taylor enseigne à ses joueurs, ce n'est pas tant l'esprit grégaire 
de la compétition sportive et la haine de l'adversaire que les idées de solidarité interpersonnelle, de soutien mutuel, de respect de la différence, de l'effort individuel au service de la réussite collective, autant de thèmes peu exploités dans la version cinématographique. Le coach n'oublie pas non plus que, pour de nombreux jeunes, la réussite sportive représente souvent un sésame pour l'obtention d'une bourse universitaire, gage d'une ascension sociale future.

Il y a là un certain paternalisme moral. Cependant, grâce à l'absence de tout manichéisme, celui-ci ne se transforme jamais en conservatisme ou en apologie réactionnaire d'un American Way of Life traditionnel ou puritain ${ }^{12}$. La façon dont Tami Taylor conseille le personnage de Becky Sprawles, au cours de la saison 4, lorsque la jeune fille tombe enceinte de façon non désirée, en témoigne : le refus de se plier à la pression de la frange néoconservatrice de Dillon conduit Tami à démissionner plutôt que d'admettre une quelconque faute morale de sa part. La ténacité de Tami contre les préjugés des militants pro-life de la ville montre que la société texane se révèle plus complexe que l'image renvoyée par les médias au cours de l'ère Bush, l'intégrité morale du personnage de Tami servant à rappeler aux téléspectateurs l'absurdité des stéréotypes géographiques et sociaux, quels qu'ils soient, y compris ceux qui concernent les habitants de ce Texas profond. De façon générale, si les Taylor sont très protecteurs, en particulier avec leur propre fille, Julie, ils sont aussi soucieux de responsabiliser les jeunes qu'ils accompagnent, dans le respect de leurs différences et de leurs choix.

Dès lors, derrière l'image du piège, la small town révèle peu à peu, grâce au format de la série qui donne du temps au récit pour se déployer pleinement, tout un ensemble de qualités susceptibles d'aider les adolescents et leur famille à se construire, à trouver un équilibre social et moral. Cette capacité de la petite ville à offrir un cadre, non pas confortable, mais plutôt rassurant et fortifiant, est particulièrement révélée par la trajectoire du personnage de Julie, la fille des Taylor,

\footnotetext{
${ }^{12}$ Les valeurs de solidarité et de responsabilité interindividuelles mises en avant dans la série peuvent néanmoins séduire les téléspectateurs de droite, à l'instar de Mitt Romney, candidat républicain défait aux élections présidentielles de 2012, qui a déclaré considérer Friday Night Lights comme l'une de ses séries favorites, reprenant même le slogan des Dillon Panthers «Clear Eyes, Full Hearts, Can’t Lose » au cours de sa campagne. On ne saurait cependant réduire les valeurs de Friday Night Lights au discours de la droite républicaine. Peter Berg, créateur de la série et soutien déclaré de Barack Obama au cours de la campagne de 2012, s'est ainsi senti obligé de dénoncer dans une lettre adressée à Mitt Romney ce qu'il interprète alors comme une tentative de récupération abusive et infondée de la part du candidat républicain puisque, selon lui, "sa politique et sa campagne ne sont clairement pas alignées sur les thèmes de notre série » («Your politics and campaign are clearly not aligned with the themes we portrayed in our series ", The Hollywood Reporter, 12 octobre 2012). Voir également la lettre ouverte de Connie Britton et de Sarah Aubrey dans USA Today en date du 28 octobre 2012 :

http://www.usatoday.com/story/opinion/2012/10/28/women-birth-control-friday-nightlights/1663895/ (dernier accès en décembre 2013).
} 
initialement rebutée à l'idée de venir s'enterrer à Dillon et qui découvre, au fur et à mesure des épisodes, les qualités insoupçonnées de la small town, au point de finir par produire un attachement très fort à la ville et à ses habitants. À la fin de la première saison, elle en arrive ainsi à ne plus vouloir quitter la petite ville pour rejoindre Austin, où son père a été recruté, expliquant comment le processus d'enracinement local a transformé sa vie et ses rêves :

JULIE TAYLOR. My opinion is that when you told me we were gonna move to Dillon, I seriously wanted to vomit. And my opinion when we got here was that every fear I had about moving... came true. And then... it changed. And I was actually happy. I've been moving around my entire life. And... for the first time, it just felt like I was at home. And I know going to Austin and being some college football coach is your dream. But I have dreams too. And they're coming true right here. (1.19)

Dans la saison 4, alors qu'elle se rend avec sa mère dans une université de Boston pour un entretien d'orientation, Julie exprime à nouveau le rapport affectif qui la lie désormais à Dillon :

JULIE. When I started high school, I couldn't wait to get out of Dillon. I thought that every book I read was like a rung on a ladder that I built to escape this town that was all about high school football and nothing else. And now that I'm actually getting close to leaving, I'm starting to appreciate that... I was shaped by my town, that I have a different viewpoint than every other person. I guess what I'm trying to say is that... I'm surprised by how happy I am to be from where I'm from. Does that make any sense? (4.8)

Loin de se réduire à un piège, l'expérience adolescente de Julie révèle comment la small town peut aussi devenir un home rassurant et attachant, propice au déploiement de sa propre personnalité et au renforcement de capacités qui lui seront utiles pour affronter l'avenir métropolitain, ouvert et incertain, que lui réserve la vie universitaire.

\section{Friday Night Lights, une alternative au pessimisme de David Simon dans The Wire}

Dans Friday Night Lights, le réalisme ne conduit donc pas à la désespérance morale. Plusieurs références intertextuelles peuvent même être interprétées comme une volonté de démarcation par rapport au discours pessimiste de nombreuses séries télévisées récentes. Ceci est particulièrement visible à partir de la quatrième saison, diffusée en 2010.

À ce moment de l'histoire, les scénaristes sont obligés de trouver de nouveaux personnages pour remplacer tous ceux qui, ayant terminé le lycée, sont partis de Dillon pour suivre un cursus à l'université (Matt, Tyra, Tim, Smash, etc.). Sans détailler les rebondissements qui ont permis aux créateurs de relancer l'intrigue et de renouveler l'intérêt des téléspectateurs, il est important de souligner 
que désormais, Eric Taylor n'est plus coach des Dillon Panthers mais est devenu celui des Lions, l'équipe de l'autre lycée de Dillon, situé à l'Est de la ville. Cet établissement est implanté au cœur d'un ghetto noir, dans des locaux vétustes, fonctionnant avec des moyens dérisoires pour accueillir des élèves majoritairement noirs, issus de familles pauvres. Ces jeunes connaissent souvent des situations familiales, économiques ou scolaires difficiles et sont beaucoup moins motivés ou disciplinés que le personnage de Smash Williams au début de la série, lui aussi issu du ghetto.

C'est dans ces conditions qu'au début du premier épisode de la quatrième saison, un officier de la police municipale de Dillon confie à Eric Taylor le soin de prendre en charge un jeune délinquant multirécidiviste, Vince Howard, menacé d'aller en prison à la moindre nouvelle infraction. Bon nombre des scènes des deux dernières saisons sont alors consacrées à suivre l'évolution de ce personnage, tiraillé entre différents modèles d'affirmation de soi (le caïd, le $Q B 1$, le fils prodigue, etc.), à suivre la façon dont le coach l'aide à se sortir des difficultés avec la police ou avec les gangsters locaux, à faire des choix responsables, à devenir un grand champion mais aussi un jeune homme digne et responsable, sur lequel sa famille et son entourage peuvent compter.

Or, le personnage de Vince est joué par Michael Bakari Jordan, un acteur resté dans la mémoire de nombreux téléspectateurs grâce au rôle tragique de Wallace qu'il incarnait dans la première saison de la série The Wire qui, elle aussi, s'intéressait à la vie des jeunes noirs des ghettos, dans la ville de Baltimore ${ }^{13}$. Le choix de cet acteur, vieilli de quelques années (voir figure 8), crée alors une résonance inattendue entre les deux séries, Friday Night Lights et The Wire.

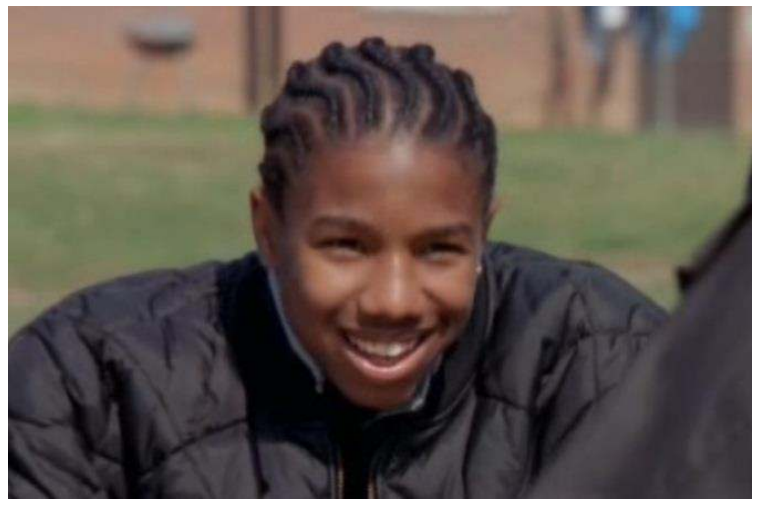

${ }_{13}$ Emmanuel Burdeau, Nicolas Vieillescazes et al., The Wire, reconstitution collective, Paris, Les prairies ordinaires/Capricci, 2011. 


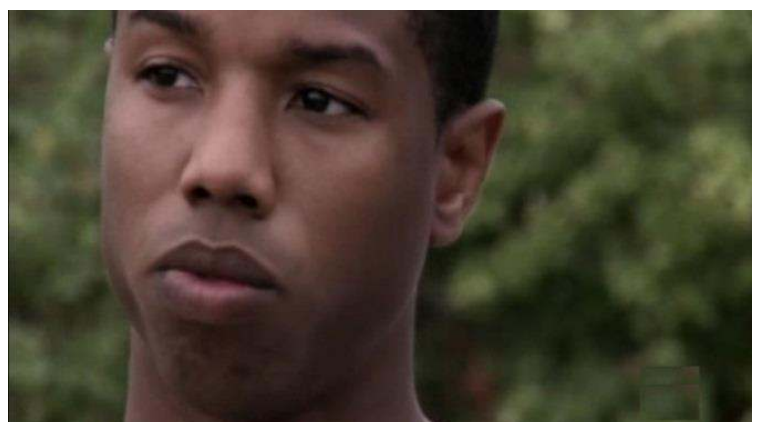

Fig. 8 : Michael Bakari Jordan interprétant Wallace dans The Wire (en haut) puis Vince dans Friday Night Lights (en bas).

La première scène dans laquelle Vince apparaît laisse d'ailleurs supposer que l'écho est voulu : on le voit poursuivi par la police alors qu'il tente de s'échapper dans le dédale des passages piétonniers d'un quartier pauvre de Dillon (voir figure 9), la scène rappelant une des nombreuses arrestations visibles dans The Wire.

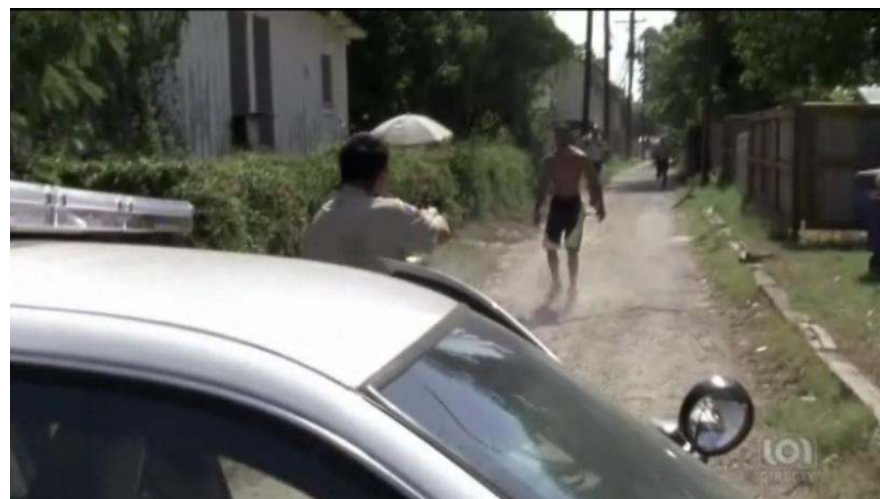

Fig. 9 : Première scène dans laquelle apparaît le personnage de Vince Howard, arrêté par la police dans une sente de Dillon qui rappelle le cadre géographique dans lequel évoluent de nombreux adolescents de Baltimore dans The Wire.

La référence à The Wire apparaît même encore plus clairement lorsque, dans l'épisode 9, le coach et Buddy Garrity cherchent à sécuriser Caroll Park, l'endroit où les jeunes d'East Dillon se réunissent en fin de journée et où un adolescent a été blessé par balle quelques jours auparavant. Ils entrent ainsi en contact avec Elden Crumpler, un ancien détenu engagé dans l'action associative auprès des jeunes. Ce 
personnage est en effet joué par Larry Gilliard Jr., acteur qui tenait le rôle de l'emblématique D'Angelo Barksdale dans la série The Wire, personnage lui aussi tragique, broyé par la logique du gang, incapable de sauver Wallace et de s'extirper de la violence urbaine de Baltimore (voir figure 10). Tout se passe alors comme si les créateurs de Friday Night Lights prenaient plaisir à faire revivre les grandes figures tragiques de la série The Wire (Wallace, D'Angelo), comme pour leur offrir une seconde vie dans laquelle, cette fois-ci, le salut et la rédemption sont possibles.
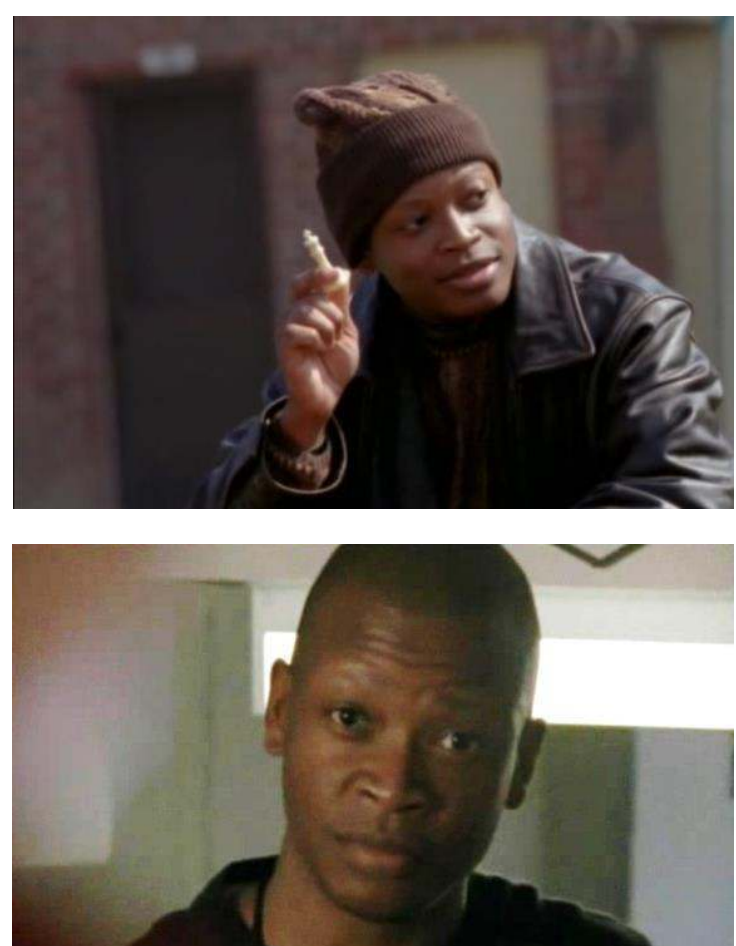

Fig. 10 : Larry Gilliard Jr. interprétant D’Angelo Barksdale dans The Wire (en haut) puis l'ancien détenu Elden Crumpler dans Friday Night Lights (en bas).

On se rappelle notamment qu'à la fin de la première saison de The Wire, Wallace est extirpé de son ghetto et envoyé par la police chez sa grand-mère qui vit à Cambridge, une petite ville du Maryland, afin d'y être mis en sécurité. Là, il ne semble trouver que de l'ennui (WALLACE. «I just got tired of that same old country scenery. It was just too slow for me ", 1.12) et décide de revenir dans son quartier pour y reprendre le « game », retour qui ne manque pas d'inspirer des soupçons aux 
chefs du gang pour lequel il travaillait jusqu'ici et qui, pour cette raison, décident de l'éliminer. Wallace trouve ainsi la mort car, malgré les mises en garde de son protecteur, D'Angelo, il est incapable de changer et de profiter de son exil rural, accablé par le tropisme tragique du ghetto : WALLACE. «I grew up in the courts, all right? I been with them niggers since forever. This is home ». Le dépaysement dans la small town n'a été pour Wallace qu'une échappée provisoire, dépourvue de sens, précédant le retour ultime dans le foyer mortel du ghetto. Au contraire de Wallace, Vince, son alter ego dans Friday Night Lights, peut compter sur l'appui du coach Taylor et de ses amis du lycée pour briser les chaînes de la reproduction sociale et éviter de se retrouver, comme son père, en prison : contrairement à The Wire, dans Friday Night Lights, la small town offre alors une alternative à la logique funeste de la ségrégation socio-spatiale.

La référence à The Wire se présente alors moins comme un hommage que comme un contrepoint décalé, Friday Night Lights rompant avec le fatalisme désabusé de David Simon dans The Wire, pour ouvrir la porte à l'espérance et à une amélioration possible des rapports humains. Dans Friday Night Lights, cette amélioration ne vient pas de l'action politique mais bien de l'engagement moral des personnages. Dans la petite ville de Dillon, Vince et le coach peuvent se croiser, cette rencontre n'étant pas traitée comme un fait contingent au milieu du chaos social, à l'image de la rencontre entre le major Bunny Colvin et le lycéen Namond Brice dans The Wire (saison 4). À la fatalité décourageante de la structure socio-spatiale des ghettos de Baltimore décrite dans The Wire par David Simon, les créateurs de Friday Night Lights opposent la possibilité d'une éthique de la sollicitude (du care) qui permettrait aux personnages de s'humaniser les uns les autres et de s'élever moralement les uns avec les autres, dans le cadre d'une small town trop exiguë pour que l'indifférence à l'égard des êtres malchanceux ou fragiles ne prolifère sans limite. Dans Friday Night Lights, le cadre géographique de la petite ville rend possible la conjonction des bonnes volontés, permettant à la communauté de continuer à exister malgré le déclin économique, les vicissitudes de l'existence ou la faiblesse morale de certains personnages.

Si la question de la réforme politique est absente de la série Friday Night Lights, la solidarité interindividuelle et la responsabilité vis-à-vis du groupe représentent des thèmes centraux qui permettent aux créateurs de souligner que, si la dignité humaine est difficile à conquérir, elle demeure toujours possible, d'une manière à la fois subtile et banale, sans déterminisme, mais sans non plus qu'une logique de happy end invraisemblable ne vienne saper l'épaisseur des drames qui se jouent au fil des épisodes. En ce sens, Friday Night Lights développe un discours véritablement humaniste, insistant sur les efforts des personnages pour adopter une attitude morale, sans que 
leurs propres fragilités face au poids des contraintes sociales ne soient édulcorées.

Avec une audience oscillant entre deux et huit millions de téléspectateurs ${ }^{14}$ et des menaces d'interruption répétées, la série Friday Night Lights n'en est pas moins parvenue à rencontrer un succès critique peu commun ${ }^{15}$, à remporter plusieurs récompenses prestigieuses et à devenir, pour une partie non négligeable de ses téléspectateurs, une série culte, attachante et inoubliable. Les choix originaux de mise en scène, le réalisme des décors, la direction subtile des acteurs, l'utilisation entêtante du post-rock gracieux et atmosphérique du groupe Explosions in the Sky, couplés à un traitement nuancé de plusieurs grands thèmes narratifs (le sport, l'amour, la famille, la pauvreté, la maladie, la délinquance, etc.) ont en effet permis aux créateurs de donner à la série une ampleur que le film n'avait pas atteinte. L'espérance réaliste qui fonde le discours humaniste de la série Friday Night Lights est ainsi traitée en soulignant qu'il ne suffit pas de désirer quelque chose pour l'obtenir. Cette perspective permet aux auteurs de la série d'insister sur le fait que si les conditionnements sociaux pèsent bien sur les individus, ils ne sont en aucune mesure une fatalité. Les personnages ont alors la possibilité de construire des existences dignes, non pas seuls ou contre les autres, comme des self-made men, mais bien grâce au soutien et à l'appui de leur entourage, grâce à la solidarité qu'offrent les petites communautés comme Dillon. Friday Night Lights se démarque ainsi d'un certain nombre de séries au ton volontairement réaliste produites au cours des années 2000 sur les chaînes du câble, que ce soit pour dénoncer les travers de la société, la brutalité des relations humaines, le poids des structures de domination ou les illusions de la politique (The Wire, HBO, 2002-2008; Deadwood, HBO, 2004-2006; Mad Men, AMC, 2007- ; Boss, Starz, 2011-; Damages, FX, Audience network, 2007-2012, etc.). Elle se démarque également des séries humoristiques dans lesquelles l'ironie ou la satire sont les seules formes d'appréciation critique possible ${ }^{16}$ (Desperate Housewives, $\mathrm{ABC}$, 2004-2012 ; Weeds, Showtime, 2005-2012 ; The Middle, ABC, 2009-).

\footnotetext{
${ }^{14}$ Voir : http://friday-night-lights.hypnoweb.net/serie/audiences.104.363/

(dernier accès en décembre 2013).

15 Voir par exemple Leah Wilson ed., A Friday Night Lights Companion, Love, Loss and Football in Dillon, Texas, Dallas, Smart Pop, 2011. En France, on notera les avis différents mais tous positifs de P. Serisier (Le Monde des Séries), A. Prié (Des séries et des hommes) ou Olivier Joyard (Les Inrocks).

${ }^{16}$ Voir Gérald Billard et Arnaud Brennetot, "Quand la critique des suburbs envahit les séries télévisées américaine », Métropolitiques, 23 novembre 2011 ; Gérald Billard, Arnaud Brennetot et al., "Subversive suburbia. L'effondrement du mythe de la banlieue résidentielle dans les séries américaines ", TV/Series, $\mathrm{n}^{\circ} 2,2012$.
} 
L'ambivalence du regard porté sur la small town par les différents personnages adolescents, mélange d'impressions de promiscuité et de sécurité, d'enfermement local et d'émancipation progressive, permet alors d'éviter toute réduction simplificatrice et de souligner le caractère contradictoire des liens sociaux qui peuvent se tisser dans une petite ville de l'Amérique profonde, crédibilisant le discours moral que veulent porter Peter Berg et Jason Katims, à savoir que l'espérance n'est pas vaine et que la dignité est accessible à tout être humain, par-delà les défaillances individuelles provisoires et les structures de domination héritées.

C'est ainsi que le téléspectateur prend plaisir à voir les personnages construire leur existence, sans que leur parcours ne soit facile, sans qu'ils soient exemptés de devoir affronter des hasards malheureux ou d'assumer des décisions lourdes de conséquences, mais sans non plus que leur trajectoire corresponde à un format unique ou à l'image stéréotypée d'une certaine idée de la réussite américaine qui consisterait, par exemple, à intégrer une université de l'Ivy League. Si beaucoup de personnages parviennent à quitter Dillon pour réaliser leur rêve ailleurs dans une grande métropole, New York, Philadelphie ou Chicago, le personnage de Tim Riggins montre que l'on peut aussi rêver d'être texan, de revivre le vieux rêve transcendantaliste, sans pour autant être un redneck stupide, misanthrope, raciste ou brutal, que l'on peut vouloir rester vivre à Dillon par amour sincère de la terre, des paysages et de l'atmosphère du Texas rural (voir figure 11). Il y a là une dimension inédite par rapport à l'ouvrage documentaire de Henry Bissinger ou à la première version cinématographique réalisée par Peter Berg.

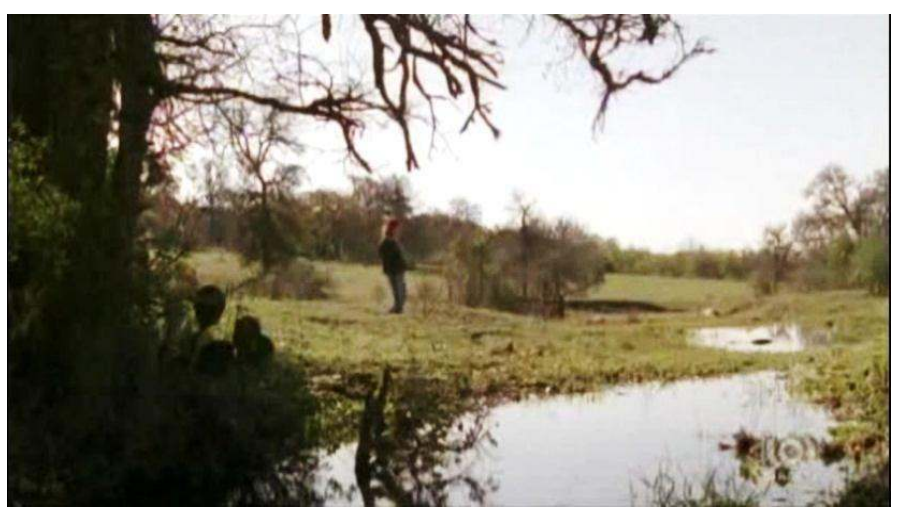

Fig. 11 : Tim Riggins, réincarnation texane du rêve transcendantaliste 
La série Friday Night Lights réinvestit alors le vieux mythe jeffersonien de la small town ${ }^{17}$, pour rappeler que le localisme ne conduit pas forcément à l'esprit de clocher ou au délire obsidional que des séries comme Jericho (CBS, 2006-2008), Deadwood ou Sons of Anarchy (FX, 2008-) ont souligné auparavant. En ce sens, Friday Night Lights rompt avec une vision négative de la small town, souvent conçue comme un bout-du-monde où la sociabilité serait forcément dégradée ${ }^{18}$. Au-delà de cette réhabilitation nuancée du localisme communautaire, la série invite aussi à rompre avec l'opposition entre small town et big city ${ }^{19}$ pour affirmer l'existence d'une complémentarité salutaire entre ces deux échelons de la hiérarchie urbaine, l'apologie de la vie locale n'aboutissant pas à la disqualification de la vie métropolitaine, les deux pouvant être bénéfiques à différents moments de l'existence de chacun.

\section{Bibliographie}

Bissinger Henry, Friday Night Lights, A Town, A Team and a Dream, Cambridge Ma, Da Capo Press, 1990.

Bissinger Henry, " Turn Out The Lights, The author of Friday Night Lights reflects on a long, resonant run ", Sports Illustrated, vol. 14, $\mathrm{n}^{\circ} 116,2011$

BILLARD Gérald et Arnaud BRENNETOT, « Quand la critique des suburbs envahit les séries télévisées américaine ", Métropolitiques, 23 novembre 2011

BILLARD Gérald et Arnaud BRENNETOT, « Le huis-clos ou l'exaltation du localisme communautaire dans les séries américaines », GRAAT OnLine, Anglophone Studies, ${ }^{\circ}$ 6, 2009.

BILlARD Gérald, Arnaud BRENNETOT et al., "Subversive suburbia. L'effondrement du mythe de la banlieue résidentielle dans les séries américaines », TV/Series, ${ }^{\circ} 2,2012$.

${ }_{17}$ Voir Catherine Maumi, Thomas Jefferson et le projet du Nouveau Monde, Paris, Éditions de La Villette, 2007.

${ }^{18}$ Voir Nicolas Roux et Jean-Michel Tixier, «Urbanités et conquête de l'Ouest. Deadwood versus La Petite maison dans la prairie », Métropolitiques, 14 novembre 2011 ; Elisabeth Tovar, "Supernatural «small-town America». Errance hantée dans les vestiges de l'Amérique industrielle », Métropolitiques, 7 novembre 2011.

19 Morton et Lucia White, The Intellectual Versus The City: From Thomas Jefferson to

Frank Lloyd Wright, Oxford, Oxford University Press, 1977. 
Burdeau Emmanuel, Nicolas VieIllescazes et al., The Wire, reconstitution collective, Paris, Les prairies ordinaires/Capricci, 2011.

FRÉMONT Armand, La Région, espace vécu, Paris, Flammarion, 1976.

Maumi Catherine, Thomas Jefferson et le projet du Nouveau Monde, Paris, Éditions de La Villette, 2007.

Monnet-CANTAgrel Hélène, " CSI, de la série au jeu : 'a more immersive experience' », TV/Series, $\mathrm{n}^{\circ} 3,2013$.

Roux Nicolas et Jean-Michel TIXIER, «Urbanités et conquête de l'Ouest. Deadwood versus La Petite maison dans la prairie", Métropolitiques, 14 novembre 2011.

TOVAR Elisabeth, «Supernatural «small-town America». Errance hantée dans les vestiges de l'Amérique industrielle », Métropolitiques, 7 novembre 2011.

Wilson Leah ed, A Friday Night Lights Companion, Love, Loss and Football in Dillon, Texas, Dallas, Smart Pop, 2011.

White Morton et Lucia, The Intellectual Versus The City: From Thomas Jefferson to Frank Lloyd Wright, Oxford, Oxford University Press, 1977. 